\title{
Second-order Sliding Mode Approaches for the Control of a Class of Underactuated Systems
}

\author{
Sonia Mahjoub $^{1} \quad$ Faiçal Mnif $^{1,2} \quad$ Nabil Derbel $^{1}$ \\ ${ }^{1}$ Advanced Control and Energy Management Laboratory, University of Sfax, \\ Sfax Engineering School, Sfax 3038, Tunisia \\ ${ }^{2}$ Department of Electrical and Computer Engineering, Sultan Qaboos University, Muscat, Oman
}

\begin{abstract}
In this paper, first-order and second-order sliding mode controllers for underactuated manipulators are proposed. Sliding mode control (SMC) is considered as an effective tool in different studies for control systems. However, the associated chattering phenomenon degrades the system performance. To overcome this phenomenon and track a desired trajectory, a twisting, a supertwisting and a modified super-twisting algorithms are presented respectively. The stability analysis is performed using a Lyapunov function for the proposed controllers. Further, the four different controllers are compared with each other. As an illustration, an example of an inverted pendulum is considered. Simulation results are given to demonstrate the effectiveness of the proposed approaches.
\end{abstract}

Keywords: Underactuated manipulator, sliding mode control, twisting algorithm, super-twisting algorithm, inverted pendulum.

\section{Introduction}

Underactuated mechanical systems (UMS) are increasingly present in the robotic field. They have less actuators than their degrees of freedom. In these systems, we find manipulators, vehicles and humanoids with several passive joints. Underactuations arise by deliberate design for the purpose of reducing the weight of the manipulator or might be caused by actuator failures. The difficulty in controlling underactuated mechanisms is due to the fact that techniques developed for fully actuated systems cannot be directly used. These systems are not feedback linearizable, yet they exhibit nonholonomic constraints and nonminimum phase characteristics ${ }^{[1]}$. Moreover, it has been shown that it is not difficult to stabilize this class of systems by continuous controllers. Because of this, the class of underactuated mechanical systems presents challenging control problems. One of the common methods used to control underactuated systems is the SMC based on Lyapunov design.

The SMC has always been considered as an efficient approach in control systems, due to its high accuracy and robustness against internal and external disturbances. The SMC approach consists of two steps. The first is to choose a manifold in the state space that forces the state trajectories to remain along it. The second is to design a discontinuous state-feedback capable of forcing the system to reach the state on the manifold in a finite time. However, the drawback of the SMC is the presence of the chattering effect, caused by the switching frequency of the control ${ }^{[2]}$.

\footnotetext{
Regular paper
Special Issue on Advances in Nonlinear Dynamics and Control

Manuscript received March 23, 2014; accepted September 25, 2014

Recommended by Guest Editor Fernando Tadeo

(C) Institute of Automation, Chinese Academy of Science and Springer-Verlag Berlin Heidelberg 2015
}

The high frequency components of the control propagating on the system can excite the unmodeled fast dynamics and therefore cause undesired oscillations. In fact, this can degrade the system performance or may even lead to instability. In the literature, three main approaches have been presented which help to reduce the chattering effects. The class of methods consist in the use of the saturation control instead of the discontinuous one. It ensures the convergence to a boundary layer of the sliding manifold. Moreover, a switching function, inside the boundary layer of the sliding manifold, was approximated by a linear feedback gain ${ }^{[3,4]}$. However, the accuracy and the robustness of the sliding mode were partially lost.

The second class of methods consist in the use of a system observer-based approach ${ }^{[5]}$. It can reduce the problem of robust control to the problem of exact robust estimation. This can lead to the deterioration of the robustness with respect to the plant uncertainties or disturbances.

Using high-order sliding mode controllers given by Levant ${ }^{[6,7]}$ as a way to reduce the chartering phenomenon and keep the main advantages of the original approach of the SMC is another way to eliminate chattering. On the other hand, the second-order sliding mode control is relatively simple to implement and it gives good robustness to external disturbances. The second-order sliding mode control (SSMC) approach can reduce the number of differentiator stages in the controller. However, the stability proofs are based usually on a geometrical or homogeneity method since the Lyapunov function is a difficult task to define $^{[7]}$. The stability and the convergence using SSMC is challenging and several trials were made to deal with those difficulties. Recently, Moreno and Osorio ${ }^{[8]}$ constructed a Lyapunov function that provides a finite time convergence, a robustness and an estimate of the convergence time for 
super-twisting algorithm. In [9], a multivariable supertwisting structure was proposed, which analyzed the stability using the ideas of Lyapunov function given in [8].

The inverted pendulum system shown in Fig. 1 is a typical benchmark of non-linear underactuated mechanical systems ${ }^{[10]}$. For this system, the control input is the force $u$ that moves the cart horizontally and the output is the angular position of the pendulum $\theta$. Therefore, the inverted pendulum has been a popular candidate to illustrate different control methods. However, despite of its simple mechanical structure, this prototype is not easy to control and requires sufficiently sophisticated control designs. Indeed, it is proven that the system is not feedback linearizable and has no corresponding constant relative degree ${ }^{[11]}$. Moreover, Zhao and Spong ${ }^{[12]}$ have shown that several geometric properties of the system are lost when the pendulum moves through horizontal positions. The objective consists of moving the cart from an initial position to a desired one and keeping the pendulum in the vertical position with a minimum of its oscillations around this position. In general, the main difficulty is to swing up the pendulum from the downward vertical position and to keep the cart stable. Numerous control techniques have been employed to stabilize the inverted pendulum such as proportional-integral-derivative (PID) controllers where the control gains are adjustable and updated online with a stable adaptation mechanism ${ }^{[13]}$.

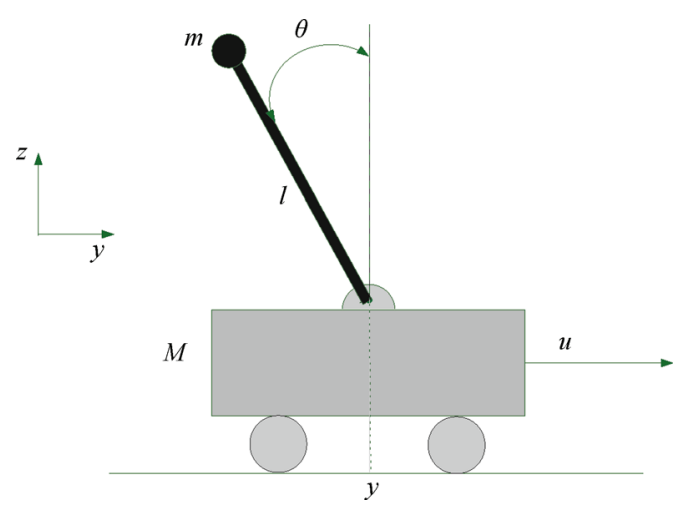

Fig. 1 Inverted pendulum

The objective of this paper is to develop a robust position tracking controller based on the first-order and the secondorder sliding mode approaches applied to an inverted pendulum. Stability of the closed loop system is carried out using candidate Lyapunov functions for the proposed controllers. The contributions of this paper are presenting the stability analysis of twisting and super-twisting controllers. Further, a modified super-twisting algorithm with simple stability analysis is proposed. This controller has almost the same propriety as the super-twisting algorithm. The paper is organized as follows. Section 2 describes the model of the inverted pendulum and the first sliding mode controller. Section 3 deals with the sliding mode controllers and the design of second-order sliding mode controllers. Section 4 discusses the simulation results of the proposed controllers.

\section{Dynamic model and control approach for an inverted pendulum}

\subsection{Dynamic model}

The dynamical behavior of an inverted pendulum can be described by the differential equations as ${ }^{[12]}$

$$
\begin{aligned}
& (m+M) \ddot{y}+m l\left(\ddot{\theta} \cos \theta-\dot{\theta}^{2} \sin \theta\right)=\tau \\
& \ddot{y} \cos \theta+l \ddot{\theta}+g \sin \theta=0
\end{aligned}
$$

where $l$ is the length of the pendulum, $m$ is the pendulum mass, $M$ is the cart mass, $\tau$ is the horizontal force action, $\theta$ is the angular deviation, and $y$ is the position of the cart which is moving horizontally.

Let $x_{1}=y, x_{2}=\dot{y}, x_{3}=\theta$ and $x_{4}=\dot{\theta}$. According to the canonical form of a class of underactuated systems, we can transform (1) into the following state space representation

$$
\begin{aligned}
& \dot{x}_{1}=x_{2} \\
& \dot{x}_{2}=f_{1}+b_{1} \tau \\
& \dot{x}_{3}=x_{4} \\
& \dot{x}_{4}=f_{2}+b_{2} \tau
\end{aligned}
$$

where $x=\left[x_{1}, x_{2}, x_{3}, x_{4}\right]^{\mathrm{T}}$ is the state variable vector, $\tau$ is the control input, $f_{1}, f_{2}, b_{1}$ and $b_{2}$ are nominal nonlinear functions, expressed as

$$
\begin{aligned}
f_{1} & =\frac{m l x_{4}^{2} \sin x_{3}-m g \sin x_{3} \cos x_{3}}{M+m \sin ^{2} x_{3}} \\
f_{2} & =\frac{(m+M) g \sin x_{3}-m l x_{4}^{2} \cos x_{3}}{l\left(M+m \sin ^{2} x_{3}\right)} \\
b_{1} & =\frac{1}{M+m \sin ^{2} x_{3}} \\
b_{2} & =\frac{-\cos x_{3}}{l\left(M+m \sin ^{2} x_{3}\right)} .
\end{aligned}
$$

Let

$$
\tau=M+m \sin ^{2} x_{3} u-\left(m l x_{4}^{2} \sin x_{3}-m g \sin x_{3} \cos x_{3}\right) .
$$

Then, (2) becomes

$$
\begin{gathered}
\dot{X}=\left[\begin{array}{c}
\dot{x_{1}} \\
\dot{x_{2}} \\
\dot{x_{3}} \\
\dot{x_{4}}
\end{array}\right]=\left[\begin{array}{c}
x_{2} \\
0 \\
x_{4} \\
\frac{g \sin x_{3}}{l}
\end{array}\right]+\left[\begin{array}{c}
0 \\
1 \\
0 \\
\frac{-\cos x_{3}}{l}
\end{array}\right] u \\
\dot{X}=f(x)+g(x) u .
\end{gathered}
$$

And (5) can be expressed by

$$
\left[\begin{array}{c}
\dot{x_{1}} \\
\dot{x_{2}} \\
\dot{x_{3}} \\
\dot{x_{4}}
\end{array}\right]=\left[\begin{array}{cccc}
0 & 1 & 0 & 0 \\
0 & 0 & 0 & 0 \\
0 & 0 & 0 & 1 \\
0 & 0 & \frac{g \sin x_{3}}{l x_{3}} & 0
\end{array}\right]\left[\begin{array}{l}
x_{1} \\
x_{2} \\
x_{3} \\
x_{4}
\end{array}\right]+\left[\begin{array}{c}
0 \\
1 \\
0 \\
\frac{-\cos x_{3}}{l}
\end{array}\right] u \text {. }
$$


Voytsekhovsky et al. ${ }^{[14,15]}$ proposed a method that can approximate the original system with an input-output linearizable control system in new coordinates. This stabilization method of nonlinear system using sliding mode control is based on coordinate transformation by mapping $T: x \mapsto \xi$ defined by

$$
\xi_{i}=L_{f}^{i-1} h(x), \quad i \in\{1,2,3,4\}
$$

with $\xi=\left(\begin{array}{llll}\xi_{1} & \xi_{2} & \xi_{3} & \xi_{4}\end{array}\right)^{\mathrm{T}}$. $T$ is defined as a local diffeomorphism with $T(0)=0$.

The dynamical system in the new coordinates can be approximated by the system model

$$
\begin{aligned}
& \dot{\xi}_{1}=\xi_{2} \\
& \dot{\xi}_{2}=\xi_{3} \\
& \dot{\xi}_{3}=\xi_{4} \\
& \dot{\xi}_{4}=L_{f}^{4}\left(T^{-1}(\xi)\right)+L_{g} L_{f}^{3} h\left(T^{-1}(\xi)\right) u
\end{aligned}
$$

where $L_{f} h(x)$ is the Lie derivative of $h(x)$ along the vector $f(x)$.

Consider the output system function defined by ${ }^{[15]}$

$$
z=h(x)=x_{1}+l \ln \left(\frac{1+\sin x_{3}}{\cos x_{3}}\right)
$$

with $\xi=T(x)$ and $T_{1}(x)=h(x)=\xi_{1}$. Define the transformation $T: x \mapsto \xi$ by

$$
T(x)=\left[\begin{array}{c}
h(x) \\
L_{f} h(x) \\
L_{f}^{2} h(x) \\
L_{f}^{3} h(x)
\end{array}\right]=\left[\begin{array}{c}
\xi_{1}=T_{1}(x) \\
\xi_{2}=T_{2}(x) \\
\xi_{3}=T_{3}(x) \\
\xi_{4}=T_{4}(x)
\end{array}\right]=\xi
$$

Then,

$$
T(x)=\left[\begin{array}{c}
x_{1}+l \ln \left(\frac{1+\sin x_{3}}{\cos x_{3}}\right) \\
x_{2}+\frac{l x_{4}}{\cos x_{3}} \\
\tan x_{3}\left(g+\frac{l x_{4}}{\cos x_{3}}\right) \\
\left(\frac{2}{\cos ^{3} x_{3}}-\frac{1}{\cos x_{3}}\right) l x_{4}^{3}+\left(\frac{3 g}{\cos ^{2} x_{3}}-2 g\right) x_{4}
\end{array}\right] .
$$

Differentiating $\xi$, we obtain

$$
\begin{aligned}
& \dot{\xi}_{1}=z^{(1)}=x_{2}+\ln \left(\frac{l x_{4}}{\cos x_{3}}\right) \\
& \dot{\xi}_{2}=z^{(2)}=\tan x_{3}\left(g+\frac{l x_{4}}{\cos x_{3}}\right) \\
& \dot{\xi}_{3}=z^{(3)}=\left(\frac{2}{\cos ^{3} x_{3}}-\frac{1}{\cos x_{3}}\right) l x_{4}^{3}+ \\
& \left(\frac{3 g}{\cos ^{2} x_{3}}-2 g\right) x_{4}-2 x_{4} \tan x_{3} u \\
& \dot{\xi}_{4}=z^{(4)}=f_{e}(x)+g_{e}(x) u
\end{aligned}
$$

where

$$
\begin{aligned}
f_{e}(x)= & \left(\frac{6 \sin x_{3}}{\cos ^{4} x_{3}}-\frac{\sin x_{3}}{\cos ^{2} x_{3}}\right) l^{4} x_{4}+\frac{6 g \sin x_{3}}{\cos ^{3} x_{3}} x_{4}^{2}+ \\
& \left(\frac{2 g \sin x_{3}}{\cos ^{3} x_{3}}-\frac{g \sin x_{3}}{\cos x_{3}}\right) 3 x_{4}^{2}+ \\
& \left(\frac{3 g}{\cos ^{2} x_{3}}-2 g\right) \frac{g \sin x_{3}}{l} \\
g_{e}(x)= & \frac{-6 x_{4}^{2}}{\cos ^{2} x_{3}}+3 x_{4}^{2}-\frac{3 g}{l \cos x_{3}}+\frac{2 g \cos x_{3}}{l} .
\end{aligned}
$$

By neglecting $2 x_{4} \tan \left(x_{3}\right)$ because this term is $o\left(x_{3}, x_{4}\right)^{2}$, we obtain a feedback linearizable nonlinear system in the state $\xi$ with

$$
\begin{aligned}
& \dot{\xi}_{1}=\xi_{2} \\
& \dot{\xi}_{2}=\xi_{3} \\
& \dot{\xi}_{3}=\xi_{4} \\
& \dot{\xi}_{4}=f_{e}(\xi)+g_{e}(\xi) u \\
& z=\xi_{1} .
\end{aligned}
$$

\subsection{First-order sliding mode controller}

Define the surface, $s=\left\{\xi \in \mathbf{R}^{4} \mid s(\xi)=0\right\}$, for $\lambda>0$,

$$
s(\xi)=\left(\frac{\mathrm{d}}{\mathrm{d} t}+\lambda\right)^{3}\left(z-z_{d}\right)
$$

We choose $z_{d}=\left[\begin{array}{llll}2 & 0 & 0 & 0\end{array}\right]^{\mathrm{T}}$. The time derivative of $s$ along the system trajectory $\xi$ is equal to

$$
\begin{aligned}
\dot{s}(\xi)= & \xi^{(4)}+3 \lambda \xi^{(3)}+3 \lambda^{2} \xi^{(2)}+\lambda^{3} \xi^{(1)}= \\
& f_{e}(\xi)+g_{e}(\xi) u+3 \lambda z^{(3)}+3 \lambda^{2} z^{(2)}+\lambda^{3} z^{(1)} .
\end{aligned}
$$

The sliding mode control is expressed by

$$
u=u_{e q}+u_{s w}
$$

where $u_{s w}$ is the switching control, $u_{e q}$ is the equivalent control yielded from $\dot{s}(\xi)=0$, and

$$
\begin{aligned}
& u_{e q}=-\frac{f_{e}(z)+3 \lambda z^{(3)}+3 \lambda z^{(3)}+\lambda^{3} z}{g_{e}(\xi)} \\
& u_{s w}=\eta \operatorname{sgn}(s)+k s
\end{aligned}
$$

where $\eta$ and $K$ are positive constants.

It is notable that for small deviations, we have $g_{e}(\xi)<$ $-3-\frac{g}{l}<0$. Choosing the Lyapunov candidate as

$$
V=\frac{1}{2} s^{2}
$$

and differentiating $V$ along the trajectories of (14) yields

$$
\dot{V}=s \dot{s}=-\eta|s|-k s^{2} \leq 0
$$

Then, the system is stable and the convergence of the sliding mode is guaranteed. 


\section{Second-order sliding mode controller}

The drawback of the first-order sliding mode control is the chattering phenomenon. As a solution to resolve this problem, a higher-order sliding mode (HOS) is proposed. In fact, HOS appears as an effective application to counteract the chattering phenomenon and the switching control signals, with higher relative degrees in a finite time ${ }^{[8,16]}$. The HOS has been introduced in [6], with the goal to get a finite time on the sliding set of order $r$ defined by $s=\dot{s}=\ddot{s}=\dddot{s}=\cdots=s^{(r-1)}=0 . \quad s$ defines the sliding variable with the $r$-th order sliding and with its $(r-1)$-th first time derivatives depending only on the state $x$. The first-order sliding mode tries to keep $s=0$. In the case of second-order sliding mode control, which only needs its measurement or evaluation of $s$, the relation should be verified as

$$
s(x)=\dot{s}(x)=0 .
$$

In the following, a twisting algorithm, a super-twisting algorithm and a modified super-twisting algorithm with a prescribed convergence law are used.

\subsection{Twisting controller}

\subsubsection{Controller approach}

Consider the sliding surface

$$
s_{1}=\left(\frac{\mathrm{d}}{\mathrm{d} t}+\lambda_{1}\right)^{2} \xi .
$$

Differentiating (22) twice gives

$$
\begin{aligned}
& \ddot{s}_{1}=f_{e}(\xi)+g_{e}(\xi) u+2 \lambda_{1} z^{(3)}+\lambda_{1}^{2} z^{(2)} \\
& \ddot{s}_{1}=\Psi(\xi)+\varphi u
\end{aligned}
$$

where $\Psi(\xi)=f_{e}(\xi)+2 \lambda z^{(3)}+\lambda^{2} z^{(2)}$ and $\varphi(\xi)=g_{e}(\xi)$.

We assume that functions $\Psi$ and $\varphi$ are bounded such that

$$
|\Psi| \leq \Psi_{d}, \quad 0<\varphi_{m} \leq \varphi \leq \varphi_{M}
$$

where $\Psi_{d}, \varphi_{m}, \varphi_{M}$ and $\varphi_{d}$ are positive scalars. Then, we have

$$
\left|\frac{\Psi}{\varphi}\right|<\frac{\Psi_{d}}{\varphi_{M}} .
$$

By imposing $\ddot{s}_{1}=0$, the equivalent control can be expressed as $u_{e q}=-\frac{\Psi}{\varphi}$.

\subsubsection{Stability analysis}

The dynamic control law using the twisting algorithm is given by ${ }^{[8]}$

$$
u_{s w}=\frac{K}{g_{e}(\xi)}\left(s_{1}+\beta \operatorname{sgn}\left(\dot{s}_{1}\right)\right)
$$

with $\beta>0,0<K \leq K_{M}$ and $K_{M}>\frac{1}{1-\beta} \frac{\Psi_{d}}{\varphi_{M}}$. The total control is defined by

$$
u=u_{e q}+u_{s w}
$$

The Lyapunov function can be chosen as

$$
V_{1}=\frac{1}{2} \lambda_{2} s_{1}^{2}+\frac{1}{2} \dot{s}_{1}^{2}
$$

Differentiating (28) yields

$$
\begin{aligned}
\dot{V}_{1}= & \lambda_{2} s_{1} \dot{s}_{1}+\dot{s}_{1} \ddot{s}_{1}= \\
& \lambda_{2} s_{1} \dot{s}_{1}+\dot{s}_{1}\left(\Psi(\xi)+g_{e}(\xi) u\right)= \\
& \dot{s}_{1}\left[\lambda_{2} s_{1}-K s_{1}-K \beta \operatorname{sgn}\left(\dot{s_{1}}\right)\right]= \\
& \dot{s}_{1} \operatorname{sgn}\left(\dot{s}_{1}\right)\left[\lambda_{2} s_{1} \operatorname{sgn}\left(\dot{s_{1}}\right)-K s_{1} \operatorname{sgn}\left(\dot{s_{1}}\right)-K \beta\right]= \\
& \left.\left|s_{1}\right|\left[\left(\lambda_{2}\left|s_{1}\right|-K\left|s_{1}\right|\right) \operatorname{sgn}\left(\dot{s}_{1} s_{1}\right)-K \beta\right)\right] \leq \\
& \left|\dot{s}_{1}\right|\left[\left(\lambda_{2}-K\right)\left|s_{1}\right|-K \beta\right] \leq 0 .
\end{aligned}
$$

Therefore, the system is stable if $\lambda_{2}-K<0$.

\subsection{Super twisting controller}

SSMC controllers require the of values of the derivatives except for the super twisting algorithm (STW). The STW is a continuous sliding mode algorithm ensuring main properties of the first-order sliding mode control for systems with Lipschitz continuous matched uncertainties or disturbances with bounded gradients ${ }^{[7]}$. It has been developed to control systems with a relative degree equal to one in order to avoid chattering.

Trajectories on the two sliding planes are characterized by twisting around the origin, but the continuous control law $u(t)$ is constituted by two terms. The first is defined by the discontinuous time derivative and the second is a continuous function of the available sliding variable ${ }^{[2]}$.

\subsubsection{Controller approach}

The derivative of the sliding surface is given as

$$
\dot{s}=f_{e}(\xi)+g_{e}(\xi) u+3 \lambda z^{(3)}+3 \lambda^{2} z^{(2)}+\lambda^{3} z^{(1)}
$$

which can be expressed as

$$
\dot{s}=\Psi_{1}(\xi)+\varphi(\xi) u
$$

where $\Psi_{1}(\xi)=f_{e}(\xi)+3 \lambda z^{(3)}+3 \lambda^{2} z^{(2)}+\lambda^{3} z^{(1)}$. The control law can be expressed by ${ }^{[16]}$

$$
u=\frac{u_{1}-\Psi_{1}(\xi)}{\varphi(\xi)}
$$

where the super twisting controller is

$$
u_{1}=-k_{1} \operatorname{sgn}(s)|s|^{\frac{1}{2}}-k_{2} s+\sigma .
$$

Variations of the term $\sigma$ are described by

$$
\dot{\sigma}=-k_{3} \operatorname{sgn}(s)-k_{4} s
$$

where $k_{1}, \cdots, k_{4}$ are positive scalars.

Substituting (32) and (33) into (31) gives

$$
\dot{s}=-k_{1} \operatorname{sgn}(s)|s|^{\frac{1}{2}}-k_{2} s+\sigma .
$$




\subsubsection{Stability analysis}

We want to prove the stability of the system with the use of Lyapunov function candidate define by

$$
V_{2}(s, z)=2 k_{3}|s|+k_{4} s^{2}+\frac{k_{5}}{2} \sigma^{2}+\gamma^{2}
$$

where $k_{5}$ is a positive scalar and

$$
\gamma=k_{1} \operatorname{sgn}(s)|s|^{\frac{1}{2}}+k_{2} s-\sigma .
$$

We introduced the positive scalar $k_{5}$ for more flexible stability conditions and for more generalization of the expression of $V_{2}$. Substituting (37) into (36) gives

$$
\begin{aligned}
& V_{2}(s, \sigma)=2 k_{3}|s|+k_{4} s^{2}+\frac{k_{5}}{2} \sigma^{2}+\left(k_{1} \frac{s}{\sqrt{|s|}}+k_{2} s-\sigma\right)^{2}= \\
& \quad \frac{1}{2|s|}\left(4 k_{3}|s|^{2}+2 k_{4} s^{2}|s|+k_{5} \sigma^{2}|s|+2 k_{1}^{2} s^{2}+\right. \\
& 4 k_{1} k_{2} s^{2} \sqrt{|s|}-4 k_{1} s \sigma \sqrt{|s|}+2 k_{2}^{2} s^{2}|s|- \\
& \left.4 k_{2} s|s| \sigma+2 \sigma^{2}|s|\right) .
\end{aligned}
$$

Define the subspace as

$$
\kappa=\left\{(s, \sigma) \in \mathbf{R}^{2} / s=0\right\} .
$$

Differentiating (38) with respect to time gives

$$
\begin{aligned}
\dot{V}_{2}(s, \sigma) & =\frac{\partial V_{2}}{\partial s} \frac{\mathrm{d} s}{\mathrm{~d} t}+\frac{\partial V_{2}}{\partial \sigma} \frac{\mathrm{d} \sigma}{\mathrm{d} t}= \\
& \frac{\partial V_{2}}{\partial s} \dot{s}+\frac{\partial V_{2}}{\partial \sigma} \dot{\sigma}= \\
& \frac{-\dot{s}}{|s|^{\frac{5}{2}}}\left(-2 k_{3}|s|^{\frac{5}{2}} \operatorname{sgn}(s)-2 k_{4} s|s|^{\frac{5}{2}}-\right. \\
& 2 k_{1}^{2} s|s|^{\frac{3}{2}}-4 k_{1} k_{2} s|s|^{2}+k_{1} k_{2} s^{2}|s| \operatorname{sgn}(s)+ \\
& 2 k_{1} \sigma|s|^{2}-k_{1} \sigma|s|^{2} s-2 k_{2}^{2} s|s|^{\frac{5}{2}}+ \\
& \left.2 k_{2}|s|^{\frac{5}{2}}+k_{1}^{2} s^{2} \operatorname{sgn}(s) \sqrt{|s|}\right)- \\
& \frac{\dot{\sigma}}{|s|}\left(-k_{5} \sigma|s|+2 k_{1} s \sqrt{|s|}+2 k_{2} s|s|-2 \sigma|s|\right) .
\end{aligned}
$$

Substituting (34) and (35) into (40) yields

$$
\begin{aligned}
& \dot{V}_{2}(s, \sigma)=\frac{-6 k_{1}^{2} k_{2} s^{2}}{|s|}+\frac{4 k_{1}^{2} \sigma s}{|s|}+\frac{k_{1}^{3} s^{4}}{|s|^{\frac{7}{2}}}-\frac{6 k_{1} k_{2}^{2} s^{2}}{\sqrt{|s|}}+ \\
& 4 k_{2}^{2} s \sigma-\frac{2 k_{1}^{3} s^{2}}{|s|^{\frac{3}{2}}}-\frac{2 k_{1} \sigma^{2}}{\sqrt{|s|}}-2 k_{2}^{3} s^{2}- \\
& 2 k_{2} \sigma^{2}+\frac{2 k_{1}^{2} k_{2} s^{4}}{|s|^{3}}-\frac{2 k_{1}^{2} s^{3} \sigma}{|s|^{3}}+\frac{8 k_{1} k_{2} s \sigma}{\sqrt{|s|}}- \\
& \frac{k_{3} k_{5} s \sigma}{|s|}-k_{5} k_{4} s \sigma-\frac{2 k_{1} k_{2} s^{3} \sigma}{|s|^{\frac{5}{2}}}+ \\
& \frac{k_{1} k_{2}^{2} s^{4}}{|s|^{\frac{5}{2}}}+\frac{k_{1} s^{2} \sigma^{2}}{|s|^{\frac{5}{2}}}
\end{aligned}
$$

Define $X=\left(|s|^{\frac{1}{2}} s \sigma\right)^{\mathrm{T}}$. Then, it is easy to show that

$$
\dot{V}_{2}(s, \sigma) \leq-\frac{1}{|s|^{\frac{1}{2}}} X^{\mathrm{T}} \Psi X-X^{\mathrm{T}} \Upsilon X
$$

where

$$
\begin{aligned}
& \Psi=\left[\begin{array}{ccc}
\Psi_{11} & 0 & \Psi_{13} \\
0 & \Psi_{22} & \Psi_{23} \\
\Psi_{31} & \Psi_{23} & \Psi_{33}
\end{array}\right] \\
& \Upsilon=\left[\begin{array}{ccc}
\Upsilon_{11} & 0 & \Upsilon_{13} \\
0 & \Upsilon_{22} & \Upsilon_{23} \\
\Upsilon_{31} & \Upsilon_{23} & \Upsilon_{33}
\end{array}\right]
\end{aligned}
$$

with

$$
\begin{aligned}
& \Psi_{11}=k_{1}^{3} \\
& \Psi_{22}=5 k_{1} k_{2}^{2} \\
& \Psi_{13}=\frac{s}{|s|}\left(\frac{k_{1} k_{5}}{2}-k_{1}^{2}\right) \\
& \Psi_{23}=-2 k_{1} k_{2} \\
& \Psi_{31}=\Psi_{13} \\
& \Psi_{32}=\Psi_{23} \\
& \Psi_{33}=k_{1}
\end{aligned}
$$

and

$$
\begin{aligned}
& \Upsilon_{11}=4 k_{1}^{2} k_{2} \\
& \Upsilon_{22}=2 k_{2}^{3} \\
& \Upsilon_{13}=-k_{1} k_{2} \frac{s}{|s|} \\
& \Upsilon_{23}=\frac{1}{2} k_{4} k_{5}-2 k_{2}^{2} \\
& \Upsilon_{32}=\Upsilon_{23} \\
& \Upsilon_{33}=2 k_{2} .
\end{aligned}
$$

Matrices $\Psi$ and $\Upsilon$ are positives definite if

$$
\begin{aligned}
k_{1}^{2} & =\frac{5}{8} k_{3} k_{5} \\
k_{2}^{2} & =2 k_{4} k_{5} .
\end{aligned}
$$

So matrices $\Psi$ and $\Upsilon$ are positive definite and consciously $\dot{V}_{2}(s, \sigma) \leq 0$. Thus, we can conclude that the system is stable.

\subsection{A modified super twisting controller}

To simplify the stability analysis, we propose a modified super twisting controller (MSTW).

\subsubsection{Controller approach}

$\dot{s}$ can be expressed as

$$
\dot{s}=\Psi_{1}(\xi)+\varphi(\xi) u=-k_{6} s+\sigma_{1} .
$$

The total controller can be expressed by

$$
u=u_{e q}+\Delta u
$$

where

$$
\Delta u=\varphi(\xi)^{-1}\left(k_{6} s+\sigma_{1}\right) .
$$

Variations of $\sigma_{1}$ are given by

$$
\dot{\sigma_{1}}=-k_{7}\left(s-k_{8} \sigma_{1}-k_{9} \operatorname{sgn} \sigma_{1}\right) .
$$

Parameters $k_{6}, k_{7}, k_{8}$ and $k_{9}$ are positive scalars. 


\subsubsection{Stability analysis}

For the stability proof, the Lyapunov function candidate is given by

$$
V_{3}\left(s, \sigma_{1}\right)=\frac{1}{2} s^{2}+\frac{1}{2 k_{7}} \sigma_{1}^{2} .
$$

Differentiating (51) with respect to time gives

$$
\begin{aligned}
& \dot{V}_{3}\left(s, \sigma_{1}\right)=s \dot{s}+\frac{1}{k_{7}} \sigma_{1} \dot{\sigma}_{1}= \\
& \quad s\left(-k_{6} s+\sigma_{1}\right)+\sigma_{1}\left(s+\frac{1}{k_{7}} \dot{\sigma}_{1}\right) .
\end{aligned}
$$

Substituting (50) into (52) gives

$$
\dot{V}_{2}\left(s, \sigma_{1}\right)=-k_{6} s^{2}-k_{8} \sigma_{1}^{2}-k_{9}|\sigma|<0 .
$$

\section{Simulation results and discussions}

Parameters of the inverted pendulum system are set as $M=20 \mathrm{~kg}, m_{0}=1.8 \mathrm{~kg}, l=0.3 \mathrm{~m}, g=9.8 \mathrm{~N} / \mathrm{kg}$. The initial conditions of the cart pendulum are $\left(y_{0}, \dot{y}_{0}\right)=(0,0)$, $\left(\theta_{0}, \dot{\theta_{0}}\right)=(0.1,0)$ and the desired position is set as $y_{d}=2$, $\theta_{d}=0$ and $\dot{y}_{d}=\dot{\theta}_{d}=0$.

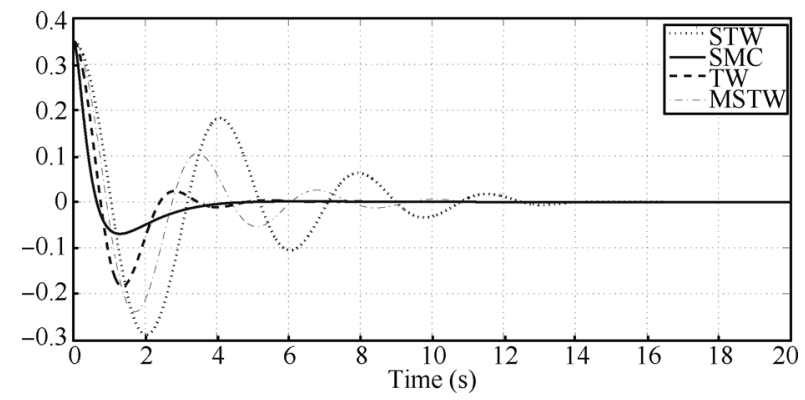

Fig. 2 Evolution of the position of $\theta$ for the uncertain system

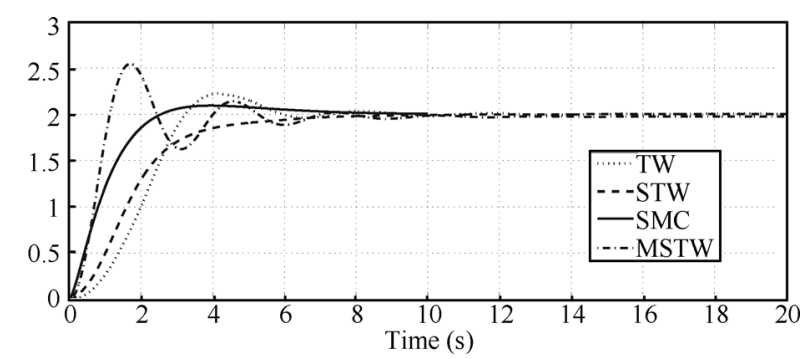

Fig. 3 Evolution of the position of $y$ for the uncertain system
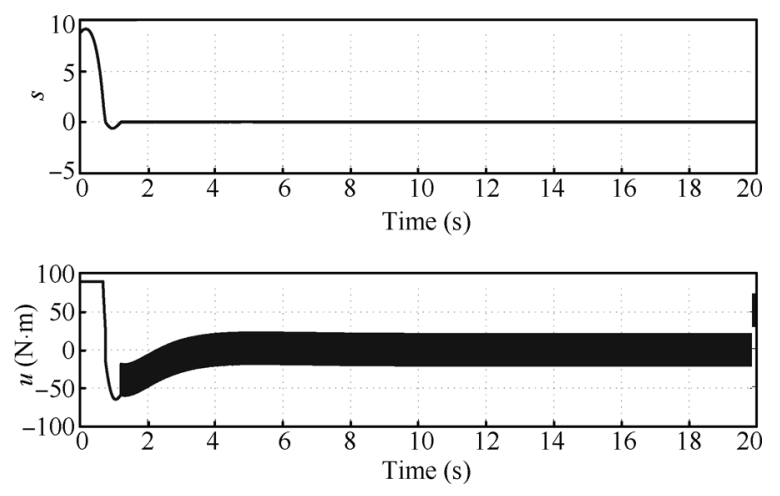

Fig. 4 Evolution of sliding surface and control by SMC
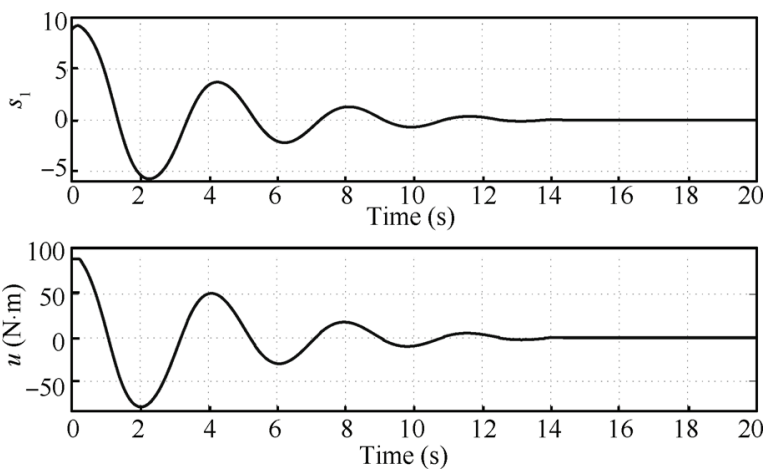

Fig. 5 Evolution of the sliding surface and the control using TW
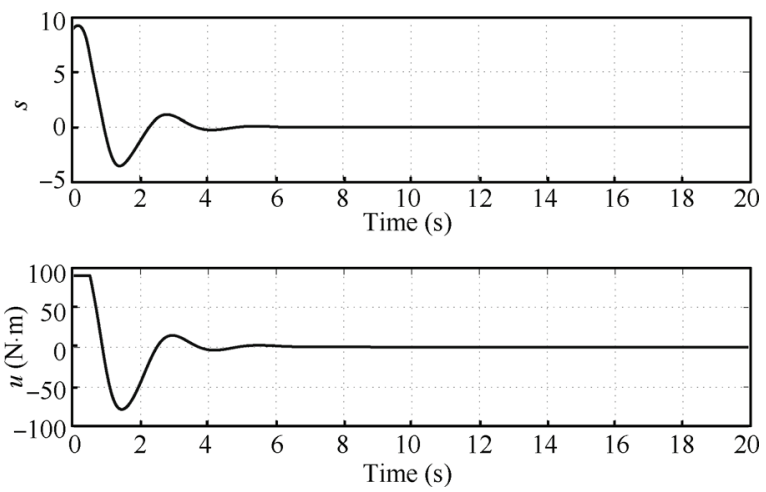

Fig. 6 Evolution of the sliding surface and the control using STW
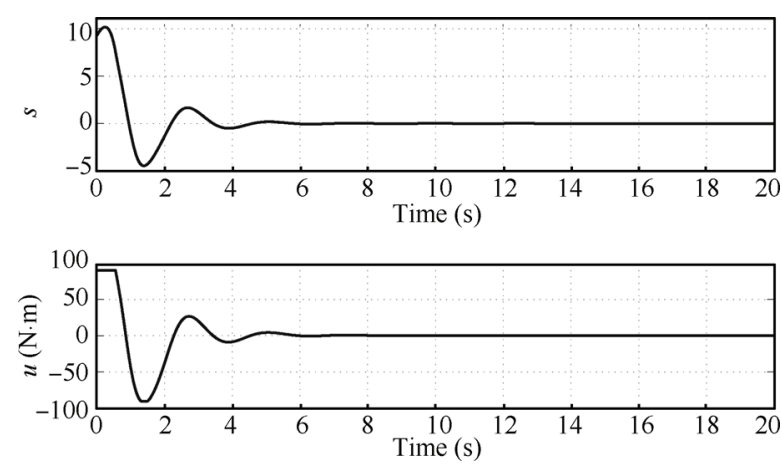

Fig. 7 Evolution of the sliding surface and the control using MSTW

Simulations are done using $\lambda=1$ and $k=20$ for the SMC, $k_{1}=40$ and $k_{2}=90$ for the twisting controller.

In Figs. 2 and 3, the simulation results for the four controllers are shown. The convergence of state variables has been established for all controllers. Furthermore, the state variables for STW and MSTW controllers converge faster than those of TW and SMC. $20 \%$ of mass uncertainties, have been considered for the pendulum and cart. We can notice the robust behaviors of the controllers with respect to parametric uncertainties. Figs. $4-7$ show that the proposed SSMC is able to compensate effectively the chatter- 
ing phenomenon better than the first-order sliding mode. Moreover, with the super-twisting controller and modified super-twisting controller, the chattering is eliminated.

For more comparisons between these approaches, we consider the following criteria

$$
J=\int_{0}^{20} u^{2} \mathrm{~d} t
$$

which is proportional to the energy delivered to the system.

It is clear from Table 1 that STW gives the least delivered energy, while the MSTW delivers the second compared to other approaches.

Table 1 Comparison of the energy criterion $\left(\times 10^{4} \mathrm{~J}\right)$

\begin{tabular}{cccc}
\hline SMC & TW & STW & MSTW \\
\hline 3.6954 & 1.5798 & 1.2624 & 1.3056 \\
\hline
\end{tabular}

\section{Conclusions}

In this paper, a second-order sliding mode controller has been designed for underactuated manipulators. This controller keep the main advantages of the original sliding mode approach and removes the chattering caused by the sliding mode approach. Simulation results of the twisting, the super-twisting and the modified super-twisting controllers show that these controllers give better performance compared to the first-order sliding mode controller. It has been shown that the proposed modified super-twisting controller have almost the same properties of the super-twisting with a simple stability analysis. Moreover, the second-order sliding-mode controller is an effective tool for the control of uncertain nonlinear systems since it overcomes the main drawback of the classical sliding-mode control approach.

\section{References}

[1] A. Isidori. Nonlinear Control Systems, Berlin, Germany: Springer Verlag, 1995.

[2] W. Perruquetti, J. P. Barbot. Sliding Mode Control in Engineering, FL, USA: CRC Press, 2002.

[3] J. A. Burton, A. S. I. Zinober. Continuous approximation of variable structure control. International Journal of Systems Science, vol. 17, no. 6, pp. 875-885, 1986.

[4] H. Lee, V. I. Utkin. Chattering suppression methods in sliding mode control systems. Annual Reviews in Control, vol. 31, no. 2, pp. 179-188, 2007.

[5] K. C. Veluvolu, Y. C. Soh, W. Cao. Robust observer with sliding mode estimation for nonlinear uncertain systems. IET Control Theory Applications, vol. 1, no. 5, pp. 1533$1540,2007$.
[6] A. Levant. Higher-order sliding modes, differentiation and output-feedback control. International Journal of Control, vol. 76, no. 9, pp. 924-941, 2003.

[7] A. Levant. Chattering analysis. IEEE Transactions on Automatic Control, vol. 55, no. 6, pp. 1380-1389, 2010.

[8] J. A. Moreno, M. Osorio. Strict Lyapunov functions for the super twisting algorithm. IEEE Transactions on Automatic Control, vol. 57, no. 4, pp. 1035-1040, 2012.

[9] I. Nagesha, C. Edwardsb. A multivariable super-twisting sliding mode approach. Automatica, vol.50, no. 3, pp. 984988, 2014.

[10] I. Fantoni, R. Lozano. Non-linear Control for Underactuated Mechanical Systems, London, UK: Springer, pp. 2142, 2002.

[11] B. Jakubczyk, W. Respondek. On the linearization of control systems. Bult. Acad. Polon. Sei. Math., vol. 28, pp. 517522,1980 .

[12] J. Zhao, M. W. Spong. Hybrid control for global stabilization of the cart pendulum system. Automatica, vol. 37, no. 12, pp. 1941-1951, 2001.

[13] W. D. Chang, R. Hwang, J. G. Chsieh. A self-tuning PID control for a class of nonlinear systems based on the Lyapunov approach. Journal of Process Control, vol. 12, no. 2, pp. 233-242, 2002.

[14] D. Voytsekhovsky, R. M. Hirschorn. Stabilization of singleinput nonlinear systems using higher-order term compensating sliding mode control. International Journal of Robust and Nonlinear Control, vol. 18, no. 4-5, pp. 468-480, 2008.

[15] C. Aguilar, R. Hirschorn. Stabilization of an Inverted Pendulum, Report on Summer Natural Sciences and Engineering Research Council of Canada, Research Project, Queens University at Kingston, Department of Mathematics and Statistics, 2003.

[16] S. V. Emel'yanov, S. V. Korovin, L. V. Levantovsky. Higher-order sliding modes in control systems. Differential Equations, vol. 29, no. 11, pp.1627-1647, 1993.

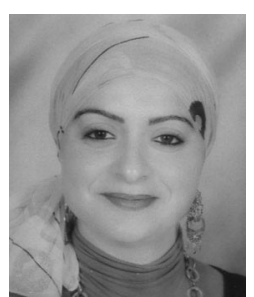

responding author)
Sonia Mahjoub received the M. Sc. degree in real time control and the $\mathrm{Ph}$. D. degree in automatic control, from the Sfax Engineering School, Tunisia in 2005 and 2013, respectively.

Her research interests include application of nonlinear control to underactuated mechanical systems.

E-mail: mahjoub_sonia@yahoo.fr (Cor-

ORCID iD: 0000-0002-3025-1754 


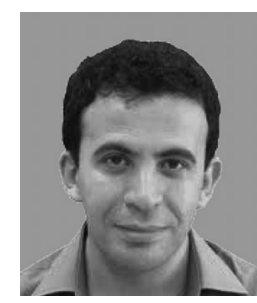

Faiçal Mnif received the B. Eng. degree in electrical engineering from Sfax Engineering School, Tunisia in 1988, the M. Sc. and the $\mathrm{Ph}$. D. degrees in control engineering from the Polytechnique School of Montreal in 1991 and 1996, respectively. From 1996 to 2000, he was with the Superior Technology in Montreal, Canada. In 2000, he joined the National Institute of Applied Sciences and Technology, where he is currently an associate professor. Since 2001, he has been on leave to Sultan Qaboos University in Oman, where he is an associate professor in Department of Electrical and Complex Mechanical Systems.

His research interests include nonlinear control theory and applications, with an emphasis on the control of complex mechanical systems.

E-mail:mnif@squ.edu.om

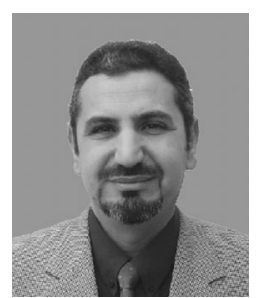

Nabil Derbel received the Engineering Diploma from the Sfax Engineering School, Tunisia in 1986, the M. Sc. degree in automatic control from the Polytechnique School of Montreal in 1986, the $\mathrm{Ph}$. D. degree from the Laboratory of $\mathrm{Au}-$ tomation and Analysis Systems of Toulouse in 1989, and the State Doctorate degree from the Sfax Engineering School, Tunisia. He joined the Tunisian University in 1989, where he held different positions in research and education. Currently, he is a full professor of automatic control at the Sfax Engineering School. $\mathrm{He}$ is an IEEE senior member.

His research interests include optimal control, complex systems, fuzzy logic, neural networks, and genetic algorithm.

E-mail: n.derbel@enis.rnu.tn 